\title{
An Experimental Study on the Electrolyte-Conditioning-Enhanced Electrokinetic Remediation of Hexavalent Chromium Contaminated Soil
}

\author{
Shanshan $\mathrm{Li}^{1}$, Jianbing $\mathrm{Li}^{2}$, Lei Liu ${ }^{3}$ \\ ${ }^{1}$ Beijing Municipal Research Institute of Environmental Protection \\ 59 Beiyingfang Street, Xicheng District, Beijing 100037, China \\ Lishanshan3496@163.com \\ ${ }^{2}$ University of Northern British Columbia, Environmental Engineering Program \\ 3333 University Way, Prince George, British Columbia, Canada V2N 4Z9 \\ Jianbing.Li@unbc.ca \\ ${ }^{3}$ Dalhousie University, Department of Civil and Resource Engineering \\ 1360 Barrington St., Halifax, Nova Scotia, Canada B3H 4R2 \\ Lei.Liu@dal.ca
}

\section{Extended Abstract}

Chromium is a commonly found heavy metal at many contaminated sites. Its high toxicity and carcinogenicity (especially at its hexavalent state) could pose serious threats to the public health and the environment. As a result, it is of great importance to develop effective remediation approaches to clean up such heavy metal contaminated soil. However, many of the conventional remediation methods suffer from some technical difficulties or economic disadvantages. In particular, the in situ remediation of heavy metal contaminated soil is often restricted by low soil permeability and high sorption capacity. In recent years, electro-kinetic remediation has been considered as a valuable alternative for enhancing heavy metal removal in low permeability soils by installing electrodes in the contaminated soil. In this method, a low direct current is applied across the electrodes, and the metal contaminants are then migrated through the combined mechanisms of electro-osmosis, electro-migration, and/or electrophoresis. However, the electrolysis reaction at the anode and cathode may adversely affect the remediation efficiency. Hydrogen ion and hydroxide ion, generated at the anode and cathode respectively, can move into the soil by electro-migration and change the $\mathrm{pH}$ of soil. The change in soil $\mathrm{pH}$ can change the chemical states of contaminants and cause them immobile. It can also change the advective transport of contaminants in soil pore fluid by electro-osmosis. Some studies on conditioning the electrolyte $\mathrm{pH}$ have been reported for improving the treatment performance of electro-kinetic remediation, but the research of this aspect in terms of hexavalent chromium $\mathrm{Cr}(\mathrm{VI})$ contaminated soil remediation is still very few. In this study, laboratory experiments were conducted to determine the feasibility of electro-kinetic remediation to treat loam soil contaminated with $\mathrm{Cr}(\mathrm{VI})$, and the electrolyte conditioning with an acidic and/or basic solution was examined on improving the treatment performance. Four sets of experiments were designed, including (a) $\mathrm{pH}$ at both anode and cathode zones was not controlled, (b) $\mathrm{pH}$ at both anode and cathode zones was maintained at 6-8, (c) pH was maintained at 6-8 at cathode, but not controlled at anode zone, and (d) pH was maintained at 6-8 at anode, but not controlled at cathode zone. Various parameters, including electric current, $\mathrm{pH}$, the removal of chromium and variation of chromium chemical fractionation were used to evaluate the remediation process. The results illustrated that the $\mathrm{Cr}(\mathrm{VI})$ and total $\mathrm{Cr}$ removal reached $98.5 \%$ and $47.6 \%$ (experiment \#a), $96.7 \%$ and $39.6 \%$ (experiment \#b), 98.7\% and 59.4\% (experiment \#c), and $99.6 \%$ and $76.4 \%$ (experiment \#d), respectively. Under the applied electric current, a partial reduction of chromium from $\mathrm{Cr}(\mathrm{VI})$ into $\mathrm{Cr}(\mathrm{III})$ was observed in soils, and this reduction may be attributed to the redox reactions that occur at the electrodes due to the electrolysis of water. In summary, electro-kinetic remediation was proved effective for chromium contaminated soil remediation, but the control of soil $\mathrm{pH}$ is very important for the success of this remediation method. The control of $\mathrm{pH}$ in the anode zone could promote the release of $\mathrm{Cr}(\mathrm{VI})$ and permits the maximum removal of chromium from soil, and thus improving the performance of electrolyte-conditioning-enhanced electro-kinetic remediation of $\mathrm{Cr}(\mathrm{VI})$ contaminated soil. 